\title{
Effects of management practices on the welfare of dairy donkeys and risk factors associated with signs of hoof neglect
}

\author{
Francesca Dai ${ }^{*}$, Giulia Segati ${ }^{1}$, Marta Brscic $^{2}$, Matteo Chincarini ${ }^{3}$, Emanuela Dalla Costa ${ }^{1}$, \\ Lorenzo Ferrari ${ }^{1}$, Faith Burden ${ }^{4}$, Andrew Judge ${ }^{4}$ and Michela Minero ${ }^{1}$ \\ 'Dipartimento di Medicina Veterinaria, Università degli Studi di Milano, 20133 Milano, Italy \\ 2 Dipartimento di Medicina Animale, Produzioni e Salute, Università degli Studi di Padova, 35020 Legnaro, Italy \\ ${ }^{3}$ Facoltà di Medicina Veterinaria, Università degli Studi di Teramo, 64100 Teramo, Italy \\ ${ }^{4}$ The Donkey Sanctuary, Sidmouth, Devon, EX10 0NU, UK
}

Received 13 March 2017; accepted for publication 11 October 2017; first published online 10 November 2017

\begin{abstract}
This Research Paper aimed to investigate donkey welfare in dairy husbandry systems and to identify the potential factors affecting it at animal level. In 2015, twelve dairy donkey farms (19-170 donkeys per farm, mean $=55 \pm 48$ ), distributed throughout Italy, were visited. On each farm, the Animal Welfare Indicators (AWIN) welfare assessment protocol for donkeys was used by two trained assessors to evaluate the welfare of animals for a total of 257 donkeys assessed. The protocol includes animal-based indicators that were entered in a digitalised system. Prevalence of different scores at individual, farm and category level were calculated. Farmers were asked to fill out a questionnaire including information regarding the management of donkeys and their final destination. Answers to the questionnaire were then considered as effects in the risk factor analysis whereas the scores of the animal-based indicators were considered as response variables. Most of the donkeys $(80 \cdot 2 \%)$ enjoyed a good nutritional status (BCS $=3$ ). $18.7 \%$ of donkeys showed signs of hoof neglect such as overgrowth and/or incorrect trimming ( $\mathrm{Min}=0 \% \mathrm{Max}=54 \cdot 5 \%$ ). Belonging to a given farm or production group influenced many of the welfare indicators. The absence of pasture affected the likelihood of having skin lesions, alopecia, low BCS scores and a less positive emotional state. Lack of routine veterinary visits $(P<0 \cdot 001)$ and having neglected hooves $(P<0.001)$ affected the likelihood of being thin $(\mathrm{BCS}<3)$. Belonging to specific production groups, lack of access to pasture and showing an avoidance reaction to an approaching human (AD) resulted in risk factors associated with a higher prevalence of signs of hoof neglect. Our results support the idea that lack of knowledge of proper donkey care among owners was behind many welfare issues found.
\end{abstract}

Keywords: Animal welfare, donkey, management, milk.

Donkey milk is a useful product for babies who suffer from multiple-allergies (cow milk, hydrolysed cow milk proteins, goat milk, soya) and have no possibility of being breastfed (lacono et al. 1992; Carroccio et al. 2000; Vincenzetti et al. 2008). Consequently, the demand for donkey milk is increasing, leading to a growing number of dairy donkey farms, especially in countries like Italy where donkey milk farming is a traditional practice. It has proven almost impossible to precisely define how many dairy donkeys there are in Italy, because the official database, held under the responsibility of the Ministry of Agricultural, Food and Forestry Policies according to the European Regulation

*For correspondence; e-mail: francesca.dai@unimi.it
$504 / 2008 / E C$, does not report the donkey specialisation (i.e. meat, milk) of the different farms. Official figures (Faostat, 2015) report that Italy is the sixth European Country in terms of donkeys farmed in the 28-EU countries after Spain, Portugal, Bulgaria, Greece and Romania. Whilst research has mainly explored the qualities of donkey milk, there is a lack of information concerning the management and welfare status of dairy donkeys. Neither national consortium nor best practice guidelines exists and there is huge variability in the professionalism of different farmers (World Horse Welfare \& Eurogroup for Animals, 2015; Dai et al. 2017). In order to ensure that this sector develops to meet the current and forecast demands for donkey milk in a sustainable way, it is paramount that animals enjoy good general management and welfare conditions (Dai et al. 2016). Concerns about possible issues affecting the 
welfare of donkeys reared for milk production have arisen (World Horse Welfare \& Eurogroup for Animals, 2015) and should be objectively addressed. Feeding practices of lactating animals and their foals, management of different animal categories (i.e. jacks, foals, etc.) on the farms, separation timing of the foals to allow milking and conditions of hooves can be assumed to be possible welfare issues for dairy donkeys. In donkeys kept for other purposes it has been reported (Dai et al. 2016) that hoof neglect is one of the major causes of concern for their welfare. Hoof neglect has a detrimental effect on long-term welfare: donkeys with neglected feet can develop chronic foot disease, which can be painful and can have a poor prognosis (Crane, 2008). Taking into consideration the implications of hoof trimming costs in the dairy donkey farm budget, we hypothesise that hoof neglect is an important animal welfare issue to address.

In 2015, the Animal Welfare Indicators project (AWIN) developed an on-farm welfare assessment protocol for donkeys (AWIN, 2015), that includes 22 animal-based indicators. An initial study was conducted on 278 donkeys of different breeds and kept for different purposes by Dai et al. (2016). No research has been conducted so far to evaluate the welfare of donkeys specifically kept for milk production using the AWIN protocol. This study aimed, therefore, to investigate donkey welfare in dairy husbandry systems and to identify the potential factors affecting it at animal level. The hypothesis behind the current investigation was that several management factors could influence animal welfare.

\section{Materials and methods}

\section{Facilities and animals}

Italian local health authorities were consulted to gather information about the presence of dairy donkey farms, the number of donkeys on each farm, and their geographical location. Twelve dairy donkey farms were selected because of their convenient accessibility, according to their geographical distribution: four in Northern Italy, four in Central Italy and four in Southern Italy. The farm managers were contacted by phone and all agreed to participate in the study on a voluntary basis. The facilities were visited between June and September 2015. All the farm managers were interested, and actively collaborated, allowing the assessors to enter the stables/paddocks and approach their donkeys. The number of donkeys per farm varied between 19 and 170 (mean $=55 \pm 48$ ). A total of 257 donkeys (Table 1$)$ of different breeds, aged between one and 360 months (mean $=65 \cdot 70 \pm$ 61.92 ) were sampled according to the AWIN welfare assessment protocol for donkeys (AWIN, 2015) and assessed.

\section{Assessors}

One veterinarian and one final-year animal science student conducted the assessments. Before carrying out the on-farm assessment, they underwent a common training period of one week, both theoretical and practical, to learn how to perform and score all the indicators by a senior veterinarian with over 10 years' experience in assessing donkey welfare. Both assessors already had some experience of donkey behaviour and welfare.

\section{Welfare assessment}

The second level of the AWIN welfare assessment protocol for donkeys (AWIN, 2015) was used to evaluate on farm the welfare of dairy donkeys (Table 2). The approximate time needed to assess a donkey varied between 5 and $10 \mathrm{~min}$. The welfare indicators covered the 4 principles and $12 \mathrm{cri}$ teria described by the Welfare Quality ${ }^{\circledR}(2009)$. In particular, the appropriate behaviour of the donkeys was assessed by recording social interaction, evidence of stereotypies and by testing the human-animal relationship with the Avoidance Distance test (AD), the Walking Down Side test (WDS) and presence of tail tuck, as described by Dalla Costa et al. (2015). Positive emotional state was assessed using Qualitative Behaviour Assessment (QBA). This method relies on the ability of humans to integrate perceived details of behaviour and enables the assessor to address the animal's experience through the observation of the expressive nature of its dynamic demeanour. The assessment was performed following procedures described by Minero et al. (2016). QBA was scored using a selected list of terms with an expressive, emotional connotation describing the different animal's expressive repertoire (Wemelsfelder, 2007).

\section{Management questionnaire}

Farmers were asked to fill in a questionnaire (detailed in Supplementary File) including information regarding the management of donkeys (nutrition, housing system, health management, milking procedures comprising training of jennies and separation of the foals) and the destination of foals and non-productive jennies (pet, work or meat production). Specific questions regarding male foals intended for meat production touched on farming methods and distances travelled to reach slaughter. Aspects derived from the questionnaire were then used as variables for the risk factor analysis. Descriptive data regarding the prevalence of different management practices in the considered sample of farms are reported elsewhere (Dai et al. 2017).

\section{Data collection and data analysis}

Data was collected using Open Data Kit application (developed by University of Washington, Department of Computer Science and Engineering), a digitised system available for Android devices (for further information see Dai et al. 2014). Data were analysed with SPSS statistical package (IBM SPSS Statistic 23) to calculate prevalence of donkeys with different scores for each welfare indicator at individual, farm and 
Table 1. Numerical consistency of animals on visited farms

$\begin{array}{lllr}\text { Animal category } & \text { Definition } & \text { Numb } \\ \text { Jenny } & \text { Lactating } & \text { Adult female donkey producing milk } & 97 \\ & \text { Dry } & \text { Non-productive adult female donkey } & 45 \\ & \text { Other } & \text { Adult female donkey kept on farm for purposes other than milk production } & 12 \\ \text { Foal } & \text { Replacement } & \text { Donkey less than 4 years old, kept on farm to became a productive animal } & 61 \\ & \text { To be sold } & \text { Donkey less than 4 years old intended to be sold to other farms or slaughterhouse } & 24 \\ \text { Jack } & & \text { Adult intact male kept on farm for reproduction } & 17 \\ \text { Gelding } & \text { Adult castrated male } & 1\end{array}$

category (e.g. Lactating, Dry, Foal) level. As for Qualitative Behaviour Assessment (QBA), the assessors used a dedicated Android application, specifically developed at Scotland's Rural College (SRUC) for automated data recording. QBA scores were downloaded from the App to an Excel file and analysed with a Principal Component Analysis (PCA, correlation matrix, no rotation). In order to test whether there were any significant effects of pasture on the QBA scores in the PCA, a one-way analysis of variance was conducted on the PC1 and PC2 scores with pasture as fixed effect and farm as random factor.

Subsequent statistical analyses of data were carried out using SAS/STAT (SAS/STAT 9.3; SAS Institute Inc., Cary, $N C, U S A)$. Data gathered through the questionnaire were considered as independent variables (factors) whereas the scores of the welfare indicators were considered as dependent variables (response), except for the Avoidance Distance Test (AD) and signs of hoof neglect that were considered as both, factor and response variables. These latter welfare indicators were considered as both factor and response, due to the fact that they are highly dependent on given managerial choices and the underlying hypothesis was that they could be considered either as direct welfare indicators of given issues or act as predisposing factors for other welfare indicators (e.g. high avoidance distance acting negatively on hoof status because a highly fearful animal may not allow hoof trimming; animals with evident signs of hoof neglect might be painful and eat less leading to a lower body condition, etc.). Descriptive statistics (Proc Freq of SAS/STAT 9.3; SAS Institute Inc., Cary, NC, USA) were carried out to assess prevalence at category and farm level of all welfare issues. Each welfare issue was submitted to one-way analysis of variance and only response variables with an average prevalence of negative scores greater than $10 \%$ at category and farm level were further submitted to risk factor analysis. Risk factor analysis was carried out at individual level for signs of hoof neglect (binary measure, presence/absence). A stepwise procedure was adopted for factor screening. Factors where a level was represented by less than $80 \%$ of the sample were excluded from further analysis. Factors retained by the regression tree (Proc C\&RT of STATISTICA, StatSoft Inc., Italy) and those significantly associated to the dependent variable for $P<0.1$ in a one-way regression analysis (Proc Logistic of SAS/STAT 9.3; SAS Institute Inc., Cary, NC, USA) were kept for further risk factor analysis. The multivariate model was built using Proc Genmod of SAS/STAT (SAS/STAT 9.3; SAS Institute Inc., Cary, NC, USA) according to the procedure described by Brscic et al. (2012). Subsets were created in case of AD data, which was split in order to analyse separately the data of foals and adult animals.

\section{Results and discussion}

\section{Effects of management practices on dairy donkey welfare}

The prevalence of donkeys affected by different welfare issues is reported in Table 2. Table 3 reports the effects of different management practices on the welfare of dairy donkeys. Only relationships that were biologically meaningful were considered, those with $P>0.1$ were excluded.

Most of the donkeys ( $80 \cdot 2 \%$ ) had a good nutritional status $(\mathrm{BCS}=3)$. When not having $\mathrm{BCS}=3$, dairy donkeys tended to be thin $(12 \cdot 8 \%$ with $\mathrm{BCS}=2)$ rather than fat $(6 \cdot 2 \%$ with $\mathrm{BCS}=4)$. Our results differ from those of a previous study carried out on European donkeys used for different purposes (Dai et al. 2016). In their sample, Dai and colleagues (2016) found that ' $25 \%$ of the assessed donkeys were moderately overweight', probably due to overfeeding. The present findings show that being overweight does not seem a relevant issue for dairy donkeys, in fact donkeys associated with dairy farming tended to be fit or moderately thin. As for our sample, production group had an effect on the nutritional status of donkeys $(P<0.001)$ : thin donkeys were mainly dry jennies $(15 \cdot 6 \%)$, foals to be sold (15\%), foals kept for replacement $(11 \cdot 7 \%)$ and donkeys kept for other purposes (40\%); while jacks tended to be moderately overweight probably due to the feeding with concentrates when combined with a lack of exercise (as reported by Dai et al. 2017). Other management practices such as a lack of routine veterinary visits $(P<0.001)$, no access to pasture $(P<0.001)$ and having neglected hooves $(P<0.001)$ affected the likelihood of being thin (Table 3). Lack of deworming treatments had a non-significant effect of lowering BCS. In general, the nutritional management of non-lactating animals (dry jennies, foals and jacks) was somehow less accurate, with huge differences between farms. Specific information about nutrient requirements for pregnant and lactating donkeys is not available in the scientific literature. However, it is recommended to feed pregnant jennies as normal in the early stages of pregnancy and then increase the energy density and quality of forages in 
Table 2. Results of welfare assessment: prevalence of donkeys affected by different welfare issues. Detailed descriptions of different scores for each welfare indicator are reported in the AWIN welfare assessment protocol for donkeys (AWIN, 2015)

Principle

Good feeding

Good health
Welfare indicator

BCS

Skin tent test

Water point

Water point cleanliness

Water point functioning

Signs of thermal stress

Shelter dimensions

Bedding

Bedding quantity

Bedding cleanliness

Integument alterations

Swollen joints

Lameness

Hair coat condition

Ocular discharge

Nasal discharges

Vulvar discharges

Faecal soiling

Abnormal breathing

Signs of hot branding

Signs of hoof neglect

Cheek palpation
Score

Prevalence in donkeys (\%)

$0 \cdot 4$

$12 \cdot 8$

$80 \cdot 2$

$6 \cdot 2$

$0 \cdot 4$

$0 \cdot 8$

93.0

$6 \cdot 2$

$58 \cdot 3$

$41 \cdot 7$

0

$82 \cdot 3$

$16 \cdot 7$

0

$58 \cdot 3$

$41 \cdot 7$

0

100

62

38

63

37

13

25

62

1

37

62

$39 \cdot 4$

50

9

$0 \cdot 8$

$0 \cdot 8$

$0 \cdot 8$

$99 \cdot 2$

0

100

$3 \cdot 5$

$96 \cdot 5$

$2 \cdot 7$

$97 \cdot 3$

$0 \cdot 4$

$99 \cdot 6$

0.4

$54 \cdot 5$

$45 \cdot 1$

$0 \cdot 8$

$99 \cdot 2$

0

100

$0 \cdot 4$

$99 \cdot 6$

18.7

$81 \cdot 3$

$0 \cdot 4$

96.9

$2 \cdot 7$ 
Table 2. (Cont.)

\begin{tabular}{|c|c|c|c|}
\hline Principle & Welfare indicator & Score & Prevalence in donkeys (\%) \\
\hline \multirow[t]{13}{*}{ Appropriate behaviour } & Stereotypies & Evidence of stereotypies & 0 \\
\hline & & No evidence of stereotypies & 100 \\
\hline & Avoidance distance & Avoidance behaviourt\$ & $21 \cdot 1$ \\
\hline & & No avoidance & 58 \\
\hline & & $\mathrm{NA}^{\dagger}$ & $20 \cdot 9$ \\
\hline & Walking down side & Negative signs ${ }^{\S \S}$ & $9 \cdot 3$ \\
\hline & & Neutral/Positive signs & $73 \cdot 9$ \\
\hline & & $\mathrm{NA}^{\dagger}$ & $16 \cdot 7$ \\
\hline & Tail tuck & Present & $6 \cdot 2$ \\
\hline & & Absent & $74 \cdot 7$ \\
\hline & & $\mathrm{NA}^{\dagger}$ & $19 \cdot 1$ \\
\hline & Social Interactions & No social contact & 6 \\
\hline & & Social contact & 94 \\
\hline
\end{tabular}

$\dagger \mathrm{NA}=$ Not Applicable.

\$Insufficient bedding = floor areas not covered by bedding are clearly visible. $\S$ Unhealthy coat $=$ dull, dry coat with or without rough coat.

I Signs of hoof neglect $=$ hooves are overgrown, rarely trimmed or trimmed incorrectly.

$\dagger \dagger$ Presence of abnormalities $=$ feeling of any asymmetry and irregular swelling.

\$Avoidance distance $=$ presence of any avoidance behaviour.

$\S \S$ Negative signs $=$ if the donkey shows any negative reaction to the movement of the observer.

Table 3. Outcomes of the one-way analyses of variance of main welfare issues

Welfare Indicator (response)

$\mathrm{BCS}=1$ or 2

$\mathrm{BCS}=4$ or 5

Presence of skin lesions

Avoidance distance

Avoidance distance (foals)

Management practices (factors)
Farm
Production group
Signs of hoof neglect
Veterinary visit frequency ${ }^{\dagger}$
Deworming
Final destination
Pasture
Production group
Production group
Deworming
Final destination
Pasture
Social interaction
Bedding $^{\dagger}$
Farm $^{\ddagger}$
Final destination $^{\S}$

D 11

\begin{tabular}{|c|c|}
\hline$\chi^{2}$ & $P$ \\
\hline $487 \cdot 51$ & $<0.001$ \\
\hline $235 \cdot 49$ & $<0.001$ \\
\hline 78.73 & $<0.001$ \\
\hline $23 \cdot 85$ & $<0 \cdot 001$ \\
\hline $2 \cdot 37$ & $0 \cdot 124$ \\
\hline $12 \cdot 87$ & 0.000 \\
\hline $154 \cdot 41$ & $<0.001$ \\
\hline $276 \cdot 03$ & $<0 \cdot 001$ \\
\hline $357 \cdot 98$ & $<0.001$ \\
\hline $1 \cdot 10$ & $0 \cdot 293$ \\
\hline $120 \cdot 73$ & $<0.001$ \\
\hline $112 \cdot 51$ & $<0.001$ \\
\hline $14 \cdot 70$ & 0.000 \\
\hline $95 \cdot 36$ & $<0.001$ \\
\hline $35 \cdot 45$ & $<0 \cdot 001$ \\
\hline $14 \cdot 55$ & $<0.001$ \\
\hline
\end{tabular}

$\uparrow$ Veterinary visit frequency = routinely planned veterinary visit vs. clinician called when necessary.

$\$$ Deworming $=$ deworming control plan applied $v s$. no deworming.

$\S$ Final destination $=$ slaughterhouse $v s$. other destination (e.g. replacement, pet, therapy, reproduction).

I Pasture $=$ access to pasture $v s$. no access to pasture.

$\dagger \dagger$ Bedding = availability of sufficient clean bedding material vs. no bedding material or insufficient/dirty bedding material.

the final trimester; this guarantees adequate supply of energy, protein, vitamins and minerals to both the mother and the foetus (Smith \& Wood, 2008; Burden, 2011). In donkeys, the detection of changes related to pathological conditions can be difficult because of the stoical nature of this species. These conditions might be accompanied only by anorexia, causing weight loss (Cox et al. 2007) and unfortuantely this can mean that, when regular veterinary checks are not scheduled, the donkey can be seriously ill before the owner realises the need to call the veterinarian (Duffield,
2008). Low body condition scores reported in our study (Table 2) could be interpreted in the light of subtle behavioural changes related to pathological conditions. Besides, BCS can be affected by parasites (Seyoum et al. 2015; Wubie \& Getaneh, 2015). Regular examination of the faeces of each donkey combined with pasture management and targeted deworming treatment, can effectively control parasites and reduce associated weight loss and development of anthelmintic resistance (Corbett et al. 2014). Our findings strengthen the indication that condition scoring 
can usefully complement the evaluation of efficacy of deworming strategies (Trawford \& Mulugeta, 2008). Access to pasture decreased the probability of being lean $(P<0 \cdot 001)$, confirming the important nutritional role of grazing as already highlighted by Smith \& Wood (2008). Another important finding was that the presence of signs of hoof neglect increased the probability of being lean $(P$ $<0 \cdot 001)$. One possible explanation could be that overgrown hooves cause difficulty of movement or painful conditions, decreasing appetite and/or the willingness to move. This result could also represent a tendency of general animal neglect: animals that received poor nutrition were also the ones less cared for within the farm.

Integument alterations affected $60 \cdot 6 \%$ of assessed donkeys; $50 \%$ of the animals presented one or more alopecic areas, 9\% presented at least one skin lesion, while only a few animals were affected by more severe alterations such as deep wounds and swellings $(0 \cdot 8$ and $0 \cdot 8 \%$ respectively). Presence of skin lesions was influenced by belonging to a given farm or production group; animals not intended for meat production had fewer skin lesions. It is likely that farmers consider animals not intended for meat production were more valuable since they usually keep them on farm for replacement or they sell them to other facilities to be involved in other activities (e.g. assisted therapy, companion animals). The animal value, as perceived by farmers, is likely to affect the level of care provided, as discussed by Boersema et al. (2013) for dairy calves rearing. Absence of sufficient clean bedding and the absence of pasture increased the likelihood of having skin lesions $(P<0 \cdot 001)$. This is probably caused by the fact that inadequate housing forces the donkeys to lie down on a hard surface (Dalla Costa et al. 2014). Finally, in the considered sample, the possibility to have full interactions with conspecifics decreased the probability of having skin lesions. Social interactions are usually reported as a risk factor for skin lesions (Pritchard et al. 2005; Mekuria \& Abebe, 2010; Dalla Costa et al. 2014), however, when social groups live in environments with an abundance of resources aggressive behaviour between donkeys might be reduced (Klingel, 1998; Moehlman, 1998; Proops et al. 2012).

General results deriving from the human-animal relationship tests were positive (Table 2). The evaluated donkeys were generally confident and not fearful of humans. However, it was not possible to perform all the behavioural tests on $20.9 \%$ of the donkeys (NA) because they were not used to be restrained with a head collar and rope. If we had tested these animals, their reaction would probably be unreliable because they were influenced by the stress of being subjected to a novel procedure. Most of these animals were foals, $80 \%$ to be sold and $31 \cdot 7 \%$ of the replacement, respectively. A lack of handling is likely to make routine husbandry, such as foot trimming and veterinary care, more difficult to undertake and cause more stress to the animal, as already demonstrated in horses by GóreckaBruzda et al. (2017). Moreover, poorly handled donkeys could be less likely to be sold as companion animals when surplus to the farmer's requirements. Improving the management procedures of these young animals could lead to adult donkeys being easier and safer to be handled. To our knowledge, this is the first time that Avoidance Distance test and Walking Down Side test were performed on foals. We considered it important to evaluate young animals since in other dairy species young animals are the less approached by stockpersons and consequently more at risk of developing stress when starting to be milked (Sutherland \& Dowling, 2014; Ivemeyera et al. 2015).

The PCA of QBA assessments for the 12 farms visited revealed five main factors with Eigen values greater than 1 ; the first three components together accounted for $69 \cdot 15 \%$ of variation between dairy donkey farms. Figure 1 shows that PC1 (30.48\% of total variance) ranged from 'agitated/aggressive' to 'at ease/relaxed'. PC2 $(24 \cdot 83 \%$ of total variance) descriptors ranged from 'friendly/playful' to 'withdrawn/apathetic'; the descriptor 'distressed' also scored highly on this component and it is difficult to explain unambiguously as it appears not to be meaningfully associated with the others. Donkeys that had access to pasture were characterised by significantly higher QBA scores on PC2 $(P<0.01)$ : animals scoring highly on this component could be described as in a more positive emotional state than donkeys without access to pasture. This finding supports the hypothesis that pasture allows animals to engage with their environment positively (Grosso et al. 2016). Studies in other species highlighted that, although animals kept extensively face a range of welfare challenges, they perceive their situation more positively than housed ones (Goddard et al. 2006; Dwyer, 2009; Sevi et al. 2010; Goddard, 2013).

\section{Risk factors associated with signs of hoof neglect}

Hoof condition appears to be a matter of concern for dairy donkey welfare: $18 \cdot 7 \%$ of the donkeys showed signs of neglect such as overgrowth and/or incorrect trimming (Table 4) with a huge variability between farms (Min $=0 \%$ Max $=54 \cdot 5 \%$ ). None of the assessed donkeys showed signs of acute laminitis. The most relevant risk factors associated with hoof neglect are listed in Table 4.

The analysis of factors associated with hoof neglect carried out in the current study suggests that the frequency of farrier visits, as declared by owners, is not a risk. This is rather surprising as it contradicts what is suggested both by common sense and the literature. As already demonstrated by researchers (Crane, 2008; Aida \& Dehghani, 2012), daily care and regular routine farriery (every six-ten weeks) are critical, and neglect of these practices predisposes to the development of foot problems. This result could be explained considering that data about the frequency of farrier visits was collected through a questionnaire because it was difficult to gather this information otherwise. An important disadvantage of questionnaires aimed at evaluating routine practices is the tendency for 


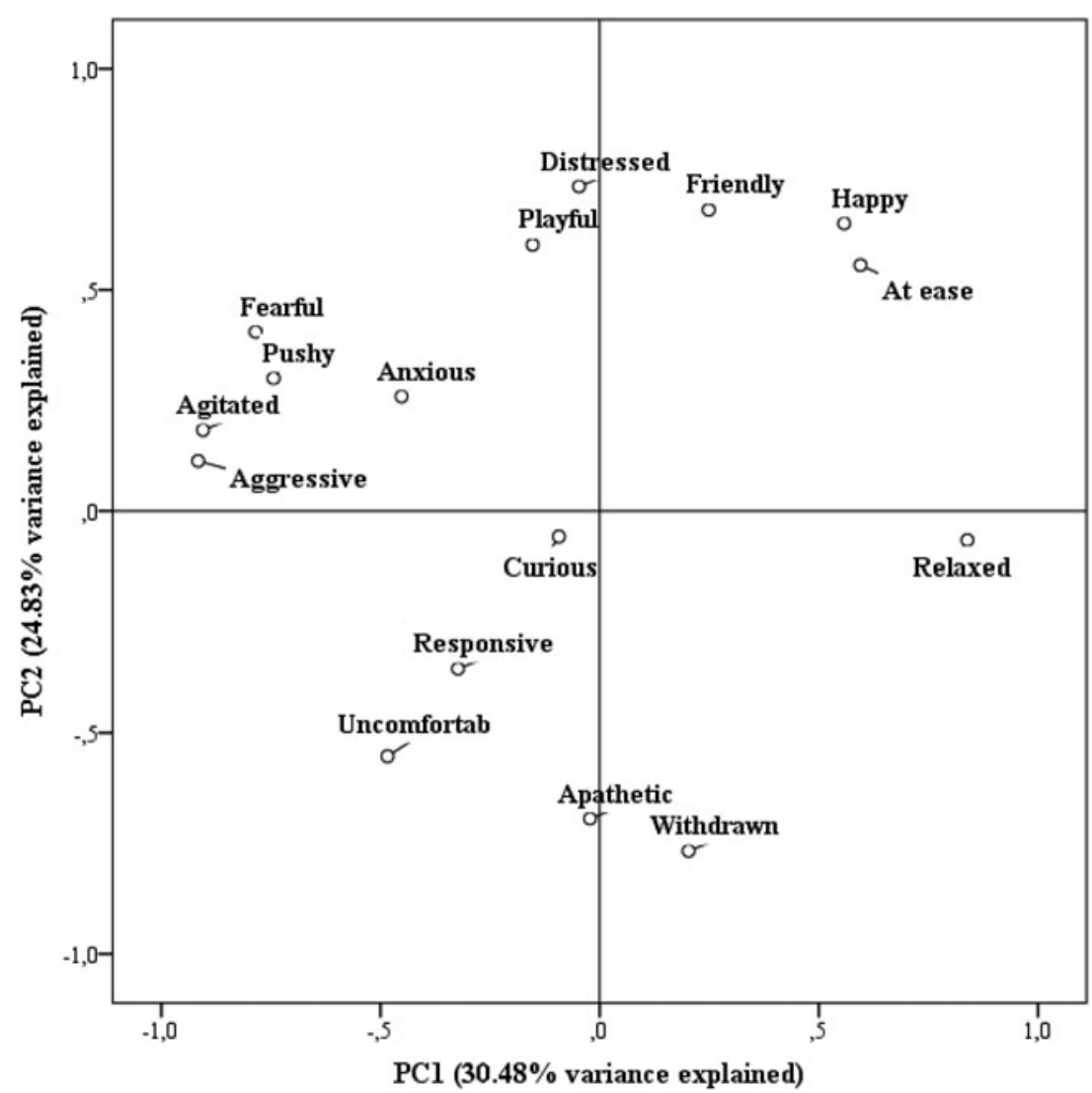

Fig. 1. Loading plot of the QBA descriptors on the first and second Principal Components (PC1 and PC2). Animals scoring high on the first component could be described as in a more positive emotional state.

Table 4. Odds ratio and 95\% confidence interval resulting from the risk factor analysis for occurrence of Signs of hoof neglect in 12 dairy donkey farms

\begin{tabular}{|c|c|c|c|c|c|}
\hline Risk factor & Levels & Prevalence $(\%)$ & Risk ratio & $95 \%$ confidence interval & $t$ pairwise comparison \\
\hline \multirow[t]{5}{*}{ Production group $(P<0 \cdot 001)$} & Dry & $21 \cdot 3$ & $2 \cdot 214$ & $1 \cdot 460-3 \cdot 357$ & $0 \cdot 000$ \\
\hline & Lactating & $22 \cdot 8$ & $2 \cdot 229$ & $1 \cdot 538-3 \cdot 231$ & $<0 \cdot 001$ \\
\hline & Jack & $35 \cdot 6$ & $2 \cdot 034$ & $1 \cdot 249-3 \cdot 312$ & 0.004 \\
\hline & Other & $17 \cdot 1$ & $1 \cdot 454$ & $0 \cdot 717-2 \cdot 950$ & $0 \cdot 300$ \\
\hline & Foal & 4 & - & - & - \\
\hline \multirow[t]{2}{*}{ Access to pasture $(P=0 \cdot 030)$} & No & 33 & $1 \cdot 361$ & $0 \cdot 779-2 \cdot 379$ & $0 \cdot 279$ \\
\hline & Yes & 17 & - & - & - \\
\hline \multirow[t]{2}{*}{ Avoidance distance $(P=003)$} & 0 & $18 \cdot 9$ & $1 \cdot 386$ & $0.979-1.963$ & 0.066 \\
\hline & 1 & $18 \cdot 6$ & - & - & - \\
\hline
\end{tabular}

people to present a favourable image of themselves. This phenomenon is called socially desirable responding and may confound results by creating false relationships between variables (van de Mortel, 2008). A possible suggestion to avoid this bias is to directly check the hoof condition, as was done in this study. When using the questionnaire in future studies, it is suggested to ask questions about routine practices in multiple ways, avoiding obvious repetitions and spacing them out.

Belonging to specific production groups, lack of access to pasture and showing an avoidance reaction to an approaching human (AD) contributed to explaining the prevalence of signs of hoof neglect. Compared to foals, all other categories of animals presented an increased risk for hoof problems. Jacks in particular had the worst conditions compared with other categories. Lack of free movement in jacks was rather common, $33.3 \%$ of farms did not allow jacks to access pasture. This could represent a possible explanation of results for hoof neglect, in fact long periods of confinement can have a negative effect on the health of the hoof (Faramarzi et al. 2009; Aida \& Dehghani, 2012). Different management solutions for jacks should be 
suggested and implemented, e.g. offering daily access to paddocks whilst minimising the risks of negative interactions with conspecifics. Another possible explanation for jacks having worse hoof conditions could be that jacks are generally considered less manageable than other animals and thus they are at higher risk of being less handled. Since incorrect management of donkey hooves could cause painful diseases (Crane, 2008), education of the owners about the correct shape of the hoof and the need for regular trimming is of paramount importance to improve donkey welfare. Owners should be encouraged to restrain and handle all of their donkeys and examine their feet on a regular basis (Crane, 2008). An increasing number of organisations within the equine sector nowadays agree that many common equine welfare problems would be effectively improved by the promotion of responsible ownership (World Horse Welfare \& Eurogroup for Animals, 2015) which includes the adoption of preventative healthcare strategies.

Access to pasture and avoidance distance meaningfully contributed to the multivariate model highlighting potential risk factors associated with poor hoof condition; however, they did not significantly increase the risk of having signs of hoof neglect. It is likely that the lack of significance in this study was affected by the high variability within the levels of these variables. Under feral conditions, donkeys spend most of their waking hours (14-16 h/day) foraging for food wandering over long distances (Crane, 2008; Smith \& Wood, 2008). In domestic donkeys, these natural conditions are difficult to replicate, however pasture could represent a valid alternative: in different species pasture was proven to favour complex natural behaviour patterns and allow exercise (Thomason, 1998; Aida \& Dehghani, 2012). Our results confirm that the human-animal relationship plays an important role in equine welfare; in fact, avoidance responses to humans were shown to be linked to hoof neglect. In the home environment, it is desirable to have donkeys that are easy to approach: the lack of confidence in or fear of humans can lead to flight reactions, which can be negatively perceived or dangerous for both donkey and human. One possible explanation for this result is that donkeys that were not used to positive human contact were likely to receive poor management treatment.

\section{Conclusions}

Donkey management can differ considerably across Europe and this is true for donkeys reared to produce milk. Our results highlight how on-farm practices influence many aspects of the welfare of dairy donkeys. In particular, belonging to a given production group influenced many of the welfare indicators such as being thin or fat, having skin lesions or showing an avoidance distance to an unknown approaching human. Management of non-lactating animals was less accurate, with huge differences between farms. One possible explanation is that lack of knowledge of proper donkey care among owners could be behind many of the welfare issues found. Targeted dissemination of information about appropriate feeding, resources, hoof care and handling of dairy donkeys would increase awareness among farmers about donkey needs and assist them in preventing welfare problems.

\section{Supplementary material}

The supplementary material for this article can be found at https://doi.org/10.1017/S0022029917000723

The authors would like to thank all the public health authorities and the farmers for their important contribution. The project 'A pilot investigation to determine welfare standards on milk/meat donkey farms in Italy and potentially influence their main drivers' has received funding from The Donkey Sanctuary, Sidmouth, Devon, EX10 0NU, UK.

\section{References}

Aida H \& Dehghani SN 2012 Hoof morphometry before and after trimming in donkeys. Research Opinions in Animal \& Veterinary Sciences 2 381-383

AWIN 2015 AWIN welfare assessment protocol for donkeys. doi:10.13130/ AWIN_DONKEYS_2015

Boersema JSC, Noordhuizen JPTM \& Lievaart JJ 2013 Hazard perception of Dutch farmers and veterinarians related to dairy young stock rearing. Journal of Dairy Science 96 5027-5034

Brscic $M$, Leruste $H$, Heutinck LFM, Bokkers EAM, Wolthuis-Fillerup $M$, Stockhofe N, Gottardo F, Lensink BJ, Cozzi G \& Van Reenen CG 2012 Prevalence of respiratory disorders in veal calves and potential risk factors. Journal of Dairy Science 95 2753-2764

Burden F 2011 Practical feeding and condition scoring for donkeys and mules. Equine Veterinary Education 24 589-596

Carroccio A, Cavataio F, Montalto G, D'Amico D, Alabrese L \& lacono G 2000 Intolerance to hydrolysed cow's milk proteins in infants: clinical characteristics and dietary treatment. Clinical and Experimental Allergy 30 1597-1603

Corbett CJ, Love S, Moore A, Burden F, Matthews JB \& Denwood MJ 2014 The effectiveness of faecal removal methods of pasture management to control the cyathostomin burden of donkeys. Parasites \& Vectors 748

Cox R, Proudman CJ, Trawford AF, Burden F \& Pinchbeck GL 2007 Epidemiology of impaction colic in donkeys in the UK. BMC Veterinary Research $\mathbf{3} 1$

Crane M 2008 The donkey's foot. In The Professional Handbook of the Donkey, pp. 188-201 (Eds. ED Svendsen, J Duncan \& D Hadrill). Yatesbury, Wiltshire, UK: Whitted Books

Dai F, Dalla Costa E, Battini M, Barbieri S, Minero M, Mattiello S \& Canali E 2014 An innovative tool for on-farm data collection and information sharing. In Proceedings of the 6th International Conference on the Assessment of Animal Welfare at Farm and Group Level, p. 153 (Eds. L Mounier \& I Veissier), Wageningen, The Netherlands: Wageningen Academic Publishers

Dai F, Dalla Costa E, Murray LMA, Canali E \& Minero M 2016 Welfare conditions of donkeys in Europe: initial outcomes from On-farm assessment. Animals 65

Dai F, Segati G, Dalla Costa E, Burden F, Judge A \& Minero M 2017 Management practices and milk production in dairy donkey farms distributed over the Italian territory. Macedonian Veterinary Review $\mathbf{4 0}$ i-vi

Dalla Costa E, Murray LAM, Dai F, Canali E \& Minero M 2014 Equine onfarm welfare assessment: a review of animal-based indicators. Animal Welfare 23 323-341

Dalla Costa E, Dai F, Murray LAM, Guazzetti S, Canali E \& Minero M 2015 A study on validity and reliability of on-farm tests to measure human-animal relationship in horses and donkeys. Applied Animal Behaviour Science $163110-121$ 
Duffield H 2008 Colic. In The Professional Handbook of the Donkey, pp. 3751 (Eds. ED Svendsen, J Duncan \& D Hadrill). Yatesbury, Wiltshire, UK: Whitted Books

Dwyer CM 2009 Welfare of sheep: providing for welfare in an extensive environment. Small Ruminant Research 86 14-21

Faostat 2015 faostat.fao.org

Faramarzi B, Thomason JJ \& Sears WC 2009 Changes in growth of the hoof wall and hoof morphology in response to regular periods of trotting exercise in standardbreds. American Journal of Veterinary Research 70 1354-1364

Goddard P, Waterhouse T, Dwyer C \& Stott A 2006 The perception of the welfare of sheep in extensive systems. Small Ruminant Research 62 215-225

Goddard PJ 2013 Small ruminant welfare: levelling the playing field or raising the bar-A European perspective. Small Ruminant Research 110 108-111

Górecka-Bruzda A, Jaworski Z, Suwała M, Sobczyńska M, Jastrzebska E, Ogłuszka M, Sankeyc, Marlena Boroń C \& Jezierski T 2017 Aversiveness of husbandry procedures for pre-weaned foals: acomparison using behavioural and physiological indices. Applied Animal Behaviour Science 191 31-38

Grosso L, Battini M, Wemelsfelder F, Barbieri S, Minero M, Dalla Costa E \& Mattiello S 2016 On-farm qualitative behaviour assessment of dairy goats in different housing conditions. Applied Animal Behaviour Science 180 51-57

Iacono G, Carrocio A, Cavataio F, Montalto G, Soresi M \& Balsamo V 1992 Use of Ass milk in multiple food allergy. Journal of Pediatric Gastroenterology and Nutrition 14 177-181

Ivemeyera S, Pisani M \& Knierim U 2015 Short-term ante-calving handling of dairy heifers in relation to heifers' behaviour and udder health after parturition. Applied Animal Behaviour Science 171 33-38

Klingel H 1998 Observations on social organization and behaviour of African and Asiatic wild asses (equus africanus and equus hemionus). Applied Animal Behaviour Science $60103-113$

Mekuria S \& Abebe R 2010 Observation on major welfare problems of equine in Meskan district, Southern Ethiopia. Livestock Research for Rural Development 2248

Minero M, Dalla Costa E, Dai F, Murray LAM, Canali E \& Wemelsfelder F 2016 Use of qualitative behaviour assessment as an indicator of welfare in donkeys. Applied Animal Behaviour Science 174 147-153

Moehlman P 1998 Feral asses (equus africanus): intraspecific variation in social organization in arid and mesic habitats. Applied Animal Behaviour Science 60 171-195
Pritchard JC, Lindberg AC, Main DCJ \& Whay HR 2005 Assessment of the welfare of working horses, mules and donkeys, using health and behaviour parameters. Preventive Veterinary Medicine 69 265-283

Proops L, Burden F \& Osthaus B 2012 Social relations in a mixed group of mules, ponies and donkeys reflect differences in equid type. Behavioural Processes 90 337-342

Sevi A, Casamassima D, Pulina G \& Pazzona A 2010 Factors of welfare reduction in dairy sheep and goats. Italian Journal of Animal Science $\mathbf{8}$ $81-101$

Seyoum Z, Tesfaye M \& Derso S 2015 Prevalence, intensity and risk factors of infestation with major gastrointestinal nematodes in equines in and around Shashemane, Southern Ethiopia. Tropical Animal Health and Production 47 1515-1521

Smith D \& Wood S 2008 Donkey nutrition. In The Professional Handbook of the Donkey, pp. 10-27 (Eds. ED Svendsen, J Duncan \& D Hadrill). Yatesbury, Wiltshire, UK: Whittet Books

Sutherland MA \& Dowling SK 2014 The relationship between responsiveness of first-lactation heifers to humans and the behavioral response to milking and milk production measures. Journal of Veterinary Behavior 9 30-33

Thomason JJ 1998 Variation in surface strain on the equine hoof wall at the mid step with shoeing, gait, substrate, direction of travel, and hoof shape. Equine Veterinary Journal Supplement 26 86-95

Trawford AF \& Mulugeta G 2008 Parasites. In The Professional Handbook of the Donkey, pp. 82-110 (Eds. ED Svendsen, J Duncan \& D Hadrill). Yaresbury, Wilshire, UK: Whitted Books

van de Mortel T 2008 Faking it: social desirability response bias in selfreport research. Australian Journal of Advanced Nursing 25 40-48

Vincenzetti S, Polidori P, Mariani P, Cammertoni N, Fantuz F \& Vita A 2008 Donkey's milk protein fractions characterization. Food Chemistry 106 640-649

Welfare Quality ${ }^{(} 2009$ Welfare Quality $\left.{ }^{(}\right)$Assessment Protocol for Cattle. Lelystad, the Netherlands: Welfare Quality ${ }^{(B)}$ Consortium

Wemelsfelder F 2007 How animals communicate quality of life: the qualitative assessment of behaviour. Animal Welfare 16 25-31.

World Horse Welfare, Eurogroup for Animals 2015 Removing the Blinkers: The Health and Welfare of European Equidae in 2015

Wubie A \& Getaneh G 2015 Study on prevalence and intensity of strongyle nematode infections in working donkeys in and around adet town, yilmana denssa woreda, Ethiopia. World Journal of Pharmaceutical Research 4 480-494 